TRANSACTIONS OF THE

AMERICAN MATHEMATICAL SOCIETY

Volume 363, Number 4, April 2011, Pages 2125-2142

S 0002-9947(2010)05230-4

Article electronically published on October 28, 2010

\title{
FOLIATIONS OF MULTIPROJECTIVE SPACES AND A CONJECTURE OF BERNSTEIN AND LUNTS
}

\author{
S. C. COUTINHO
}

To Israel Vainsencher on his 60th birthday

\begin{abstract}
We use foliations of multiprojective spaces defined by Hamiltonian functions on the underlying affine space to prove the three dimensional case of a conjecture of Bernstein and Lunts, according to which the symbol of a generic first-order differential operator gives rise to a hypersurface of the cotangent bundle which does not contain involutive conical subvarieties apart from the zero section and fibres of the bundle.
\end{abstract}

\section{INTRODUCTION}

Although this paper is concerned with foliations on multiprojective spaces induced by a Hamiltonian function of the underlying affine space, its motivation lies in the theory of $\mathcal{D}$-modules, and it is with this theory that we begin.

A much studied mathematical object is the ring of differential operators of the polynomial algebra in $n$ variables over the complex numbers. This ring, known as the Weyl algebra, and denoted by $A_{n}$, has a very interesting representation theory. Our point of departure is the Bernstein inequality, according to which the Gelfand-Kirillov dimension of a finitely generated $A_{n}$-module cannot be less than $n$. The $A_{n}$-modules with dimension exactly $n$ are called holonomic and have been intensively studied because of their many applications in representation theory, the theory of partial differential equations, and various other areas of mathematics; see, for example, 4, [5] and [12.

For a time, in the early 1980s, some experts believed that all irreducible $A_{n^{-}}$ modules were holonomic. This was disproved by J. T. Stafford in 1985 with an explicit construction of an irreducible $A_{n}$-module of dimension $2 n-1$, for all $n \geq 2$; see [17. In 1988, J. Bernstein and V. Lunts described a geometric construction of whole families of nonholonomic irreducible modules over the Weyl algebra. In fact, they gave two different constructions, the second of which is the one that concerns us here; see [3, p.236ff].

Received by the editors July 6, 2009

2000 Mathematics Subject Classification. Primary 37F75, 16S32; Secondary 37J30, 32C38, $32 \mathrm{~S} 65$.

Key words and phrases. Derivation, singularity, invariant variety, Hamiltonian, symplectic geometry, $\mathcal{D}$-module.

The author wishes to thank Jorge Vitório Pereira for his help with section 4. The work on this paper was partially supported by a grant from CNPq.

(C)2010 American Mathematical Society Reverts to public domain 28 years from publication 
Both Stafford's construction and the second one in the paper by Bernstein and Lunts proceed by adding a carefully chosen polynomial to a derivation of the polynomial ring. The resulting first-order differential operator of $A_{n}$ generates a maximal left ideal, and the quotient module has Gelfand-Kirillov dimension $2 n-1$. Thus, if $n \geq 2$, it cannot be holonomic.

The derivation used by Stafford is very special and has no singularities in $\mathbb{C}^{n}$. Bernstein and Lunts, on the other hand, start from a generic derivation, which will be necessarily singular. While the proof that Stafford's example works is computational, the one by Bernstein and Lunts is geometric and depends on the nonexistence of certain subvarieties (called involutive conical) of the hypersurface defined by the principal symbol of a generic derivation in the cotangent bundle $T^{*} \mathbb{C}^{n}$. There is a glitch, though. They were able to prove this nonexistence result only for $n=2$. See section 7 for more background and a detailed statement of the conjecture.

The aim of this paper is to extend our knowledge of the conjecture of Bernstein and Lunts by proving it for $n=3$. In order to do this we projectivize the cotangent bundle $T^{*} \mathbb{C}^{3} \cong \mathbb{C}^{3} \times \mathbb{C}^{3}$ as $\mathbb{P}^{3} \times \mathbb{P}^{2}$ by taking into account that the varieties that we are dealing with are conical, that is, homogeneous in the fibres of $T^{*} \mathbb{C}^{3}$. The symbol of a derivation gives rise to a Hamiltonian function $f$ that is linear in these fibres. The corresponding Hamiltonian vector field determines a foliation of the multiprojective space. The core of the paper is dedicated to showing that the hypersurface defined in $\mathbb{P}^{3} \times \mathbb{P}^{2}$ by the homogenization of $f$ does not have any invariant subvarieties of codimension one, apart from $H_{\infty} \times \mathbb{P}^{2}$, where $H_{\infty}$ is the hyperplane at infinity of $\mathbb{P}^{3}$.

The paper is divided into seven sections. Section 2 contains a number of elementary results of commutative algebra that will be used throughout the paper. In section 3, besides establishing the notation to be used in the paper, we introduce foliations in multiprojective spaces and define those that are induced from Hamiltonian functions. The proofs of the main results are split between section 5 , which contains two key lemmas, and sections 4 and 6. which contain the proofs of the theorems on the nonexistence of invariant subvarieties. Finally, section 7 begins with a description of the background to the conjecture of Bernstein and Lunts and ends with a proof of the conjecture for $n=3$.

\section{Preliminaries}

If $A$ is a commutative ring, we denote by $A[\mathbf{x}]$ the polynomial ring $A\left[x_{1}, \ldots, x_{n}\right]$.

2.1. Prime ideals. We begin with some criteria under which certain ideals of a polynomial ring are prime. The following notation will be in force until the end of the section:

$m \geq 2$ is an integer, $a_{1}, \ldots a_{m}$ are elements of a domain $A$ and

$$
f=a_{1} y_{1}+\cdots+a_{m} y_{m} \in A[\mathbf{y}]=A\left[y_{1}, \ldots, y_{m}\right] .
$$

Proposition 2.1. Let $m=2$. If $\left(a_{1}\right)$ is a radical ideal of $A$ and $a_{2}$ does not belong to any of the primes minimal over $\left(a_{1}\right)$, then $(f)$ is saturated in $A\left[y_{1}, y_{2}\right]$ with respect to the multiplicative set of powers of $a_{1}$.

Proof. First of all, $\{f\}$ is a Gröbner basis of $(f)$ in $A\left[y_{1}, y_{2}\right]$ with respect to the lexicographic order with $y_{1}>y_{2}$. Therefore, by [1, Proposition 4.4.4, p. 240] the 
desired result will follow if we prove that

$$
(f)^{e} \cap A\left[y_{1}, y_{2}\right]=(f),
$$

where $(f)^{e}$ denotes the extension of $(f)$ to the ring $A_{a_{1}}$, which is the localization of $A$ at the powers of $a_{1}$. Suppose, by contradiction, that this is false. Then, there exists $g \in A\left[y_{1}, y_{2}\right]$ such that

$$
a_{1}^{-1} g f \in A\left[y_{1}, y_{2}\right] \text { and } a_{1}^{-1} g \notin A\left[y_{1}, y_{2}\right] \text {. }
$$

In particular, at least one of the monomials of $g$ has a coefficient, which we denote by $c$, such that $a_{1}^{-1} c \notin A$. On the other hand, (2.1) implies that $g f \in A\left[y_{1}, y_{2}\right] a_{1}$; so $c a_{2} \in\left(a_{1}\right)$, which is where we need to assume that $m=2$. Thus, it follows that $c \in\left(a_{1}\right)$, because $\left(a_{1}\right)$ is radical and $a_{2}$ is not contained in any of the primes minimal over $\left(a_{1}\right)$. But this means that $a_{1}^{-1} c \in A$, which is a contradiction.

Corollary 2.2. Let $m=3$ and $n \geq 2$ be integers and consider the ring $A=$ $\mathbb{C}\left[x_{1}, x_{2}, \ldots, x_{n}, y_{1}, y_{2}, y_{3}\right]$. If $a_{1}$ is irreducible, $\left(a_{1}, a_{2}\right)$ is a radical ideal of $A$ and $a_{3}$ is not contained in the primes minimal over $\left(a_{1}, a_{2}\right)$, then the ideal $\left(a_{1}, a_{2} y_{2}+a_{3} y_{3}\right)$ is prime in $A$.

Proof. Since $\left(a_{1}\right)$ is a radical ideal of $A$, the $\operatorname{ring} D=A /\left(a_{1}\right)$ is a domain. Denoting by $\bar{g}$ the image in $D\left[y_{1}, y_{2}, y_{3}\right]$ of an element $g \in A$, we have that if

$$
I=\left(a_{1}, a_{2} y_{2}+a_{3} y_{3}\right)
$$

then

$$
\bar{I}=\left(\overline{a_{2} y_{2}+a_{3} y_{3}}\right)
$$

in $D[\mathbf{y}]=D\left[y_{1}, y_{2}, y_{3}\right]$. Let $S$ be the multiplicative set of powers of $\bar{a}_{2}$ and denote by $Q$ the ring of fractions of $D[\mathbf{y}]$ with respect to $S$. By Proposition 2.1] $\bar{I}$ is saturated in $D\left[y_{1}, y_{2}, y_{3}\right]$. Hence, the natural map

$$
D[\mathbf{x}] / I \rightarrow Q[\mathbf{x}] / Q \bar{I}
$$

is injective. However, the image of $y_{2}+a_{3} y_{3} / a_{2}$ is a generator of $Q \bar{I}$ over $Q$, so that

$$
D[\mathbf{x}] / I \rightarrow Q[\mathbf{x}] / Q \bar{I} \cong Q\left[y_{3}\right]
$$

which is a domain. Hence, $D[\mathbf{x}] / \bar{I}$ is itself a domain, which implies that $\bar{I}$ is a prime ideal of $D[\mathbf{x}]$. Therefore, $I$ is prime ideal, as we wished to prove.

For the next two results we also assume that $A=\mathbb{C}\left[x_{1}, \ldots, x_{n}\right]$ and that $a_{1}, \ldots a_{m}$ are polynomials of degree $k \geq 2$. A polynomial which is homogeneous with respect to the $x$ variables and also homogeneous with respect to the $y$ variables is called bihomogeneous.

Proposition 2.3. If $\left(a_{1}\right)$ is a prime ideal of $\mathbb{C}[\mathbf{x}]$ and $a_{2} \notin\left(a_{1}\right)$, then $f$ is irreducible in $\mathbb{C}[\mathbf{x}, \mathbf{y}]$.

Proof. The hypotheses imply that $\operatorname{gcd}\left(a_{1}, a_{2}\right)=1$. However, $f$ has degree one as a polynomial in the $y$ s, so any factorization of $f$ will have to be of the form $f=c \hat{f}$ for some $c \in \mathbb{C}[\mathbf{x}]$. But this implies that $c$ divides $\operatorname{gcd}\left(a_{1}, a_{2}\right)=1$. Therefore, $c \in \mathbb{C}$ and $f$ is irreducible.

Proposition 2.4. If $m \leq n$ and $a_{1}, \ldots, a_{m}$ are homogeneous, then $h=\sum_{i=1}^{m} x_{i} y_{i}$ is irreducible modulo $f$. 
Proof. Suppose, by contradiction, that $h \equiv g_{1} g_{2}(\bmod f)$, where $g_{1}, g_{2} \in \mathbb{C}[\mathbf{x}, \mathbf{y}]$. Thus, $h=g_{1} g_{2}+f q$, for some $q \in \mathbb{C}[\mathbf{x}, \mathbf{y}]$. Since $h$ and $f$ are bihomogeneous, we may assume that so are $g_{1}, g_{2}$ and $q$. However, $h$ has total degree 2 , while the total degree of $f$ is at least 3. Therefore, $q=0$ and $h=g_{1} g_{2}$. But $h$ is irreducible in $\mathbb{C}[\mathbf{x}, \mathbf{y}]$, so either $g_{1}$ or $g_{2}$ has to be constant. Hence, $h$ is irreducible modulo $f$.

2.2. Factorial domains. We now proceed to prove that some rings that appear in later sections have the property of unique factorization into primes.

Theorem 2.5. Let $A=\mathbb{C}\left[x_{1}, \ldots, x_{n}\right]$, and assume that:

(1) $m=3$;

(2) $\left(a_{1}\right)$ is a prime ideal of $A$;

(3) $a_{3}$ does not belong to the primes minimal over $\left(a_{1}, a_{2}\right)$.

Then, $A[\mathbf{y}] /(f)$ is a factorial ring.

Proof. By Proposition 2.3, $f$ is prime in $A[\mathbf{y}]$; so $B=A[\mathbf{y}] /(f)$ is a domain. But by Nagata's factoriality lemma, if $B_{a_{1}}$ is a factorial domain and $a_{1}$ is prime in $B$, then $B$ is factorial; see [16. Théoréme 5, p. 31] or [9. Lemma 19.20, p. 487]. However,

$$
B_{a_{1}} \cong A_{a_{1}}\left[y_{2}, y_{3}\right]
$$

is a factorial domain by [16. Théoréme 4, p. 29 and Corollaire 1, p. 23]. On the other hand, $\left(a_{1}, f\right)=\left(a_{1}, a_{2} y_{2}+a_{3} y_{3}\right)$ is a prime ideal by Corollary 2.2. Therefore,

$$
B /\left(a_{1}\right) \cong A\left[y_{2}, y_{3}\right] /\left(a_{1}, a_{2} y_{2}+a_{3} y_{3}\right)
$$

is a domain. Hence, $\left(a_{1}\right)$ is prime in $B$ and the desired result follows.

Corollary 2.6. If $m=3$ and $h=\sum_{i=1}^{m} x_{i} y_{i}$, then $\mathbb{C}[\mathbf{x}, \mathbf{y}] /(h)$ is a factorial domain.

Proof. We need only check that the hypotheses of Theorem 2.5 hold for $h$. However, the coefficient of $y_{1}$ in $h$ is $x_{1}$, which is clearly irreducible in $\mathbb{C}[\mathbf{x}]$. On the other hand, $\left(x_{1}, x_{2}\right)$ is prime and does not contain $x_{3}$, so (3) also holds.

\section{Foliations}

In this section we collect a number of results on foliations that are used later in the paper. The notation that will be in force throughout the paper is established in 3.3

3.1. Basic definitions. We briefly review those basic definitions from the theory of holomorphic foliations that will be required in the paper. Let $X$ be a smooth complex projective variety of dimension at least 2. A foliation (of dimension one) on $X$ is an $\mathcal{O}_{X}$-homomorphism

$$
\xi: \Omega_{X}^{1} \rightarrow \mathcal{L},
$$

where $\mathcal{L}$ is a line bundle over $X$ and $\Omega_{X}^{1}$ is the sheaf of Kähler differentials over $X$. The foliation $\xi$ can also be defined by

(1) the $\mathcal{O}_{X}$-homomorphism $\xi^{\vee}: \mathcal{L}^{\vee} \rightarrow \Theta_{X}$ or

(2) a global section of $\Theta_{X} \otimes \mathcal{L}$, 
where $\mathcal{L}^{\vee}=\mathcal{H}^{\circ} m_{\mathcal{O}_{X}}\left(\mathcal{L}, \mathcal{O}_{X}\right)$. We swap between these definitions, whenever needed, without further comment. Moreover, we do not always distinguish between a foliation and the map or section $\xi$ that is used to define it.

A singularity of $\xi$ is a point $x \in X$ such that $\xi$ is not surjective at $x$. Equivalently, $x \in X$ is a singularity of $\xi$ if $\Theta_{X} / \operatorname{Im}\left(\xi^{\vee}\right)$ is not free at $x$. The set of all singularities of $\xi$ is denoted by $\operatorname{Sing}(\xi)$. A subscheme $Y$ of $X$ is invariant under $\xi$ if there exists a map $\left.\Omega_{Y}^{1} \rightarrow \mathcal{L}\right|_{Y}$ such that the diagram

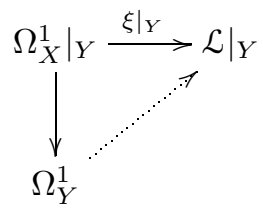

is commutative. For more details see [7].

3.2. Multiprojective spaces. We now turn to the case $X=\mathbb{P}^{m} \times \mathbb{P}^{n}$. For $j=1,2$, let $\pi_{j}$ be the projection on the $j$ th component of $X$. A line bundle $\mathcal{L}$ of $\mathbb{P}^{m} \times \mathbb{P}^{n}$ has the form

$$
\mathcal{L}=\pi_{1}^{*}\left(\mathcal{O}_{\mathbb{P}^{m}}(r)\right) \otimes_{\mathcal{O}_{X}} \pi_{2}^{*}\left(\mathcal{O}_{\mathbb{P}^{n}}(s)\right)
$$

for integers $r$ and $s$, and

$$
\Theta_{\mathbb{P}^{m} \times \mathbb{P}^{n}} \cong \pi_{1}^{*}\left(\Theta_{\mathbb{P}^{m}}\right) \oplus \pi_{2}^{*}\left(\Theta_{\mathbb{P}^{n}}\right) .
$$

Therefore, a foliation $\xi$ of $\mathbb{P}^{m} \times \mathbb{P}^{n}$ is a global section of the sheaf

$$
\left(\pi_{1}^{*}\left(\Theta_{\mathbb{P}^{m}}(r)\right) \otimes_{\mathcal{O}_{X}} \pi_{2}^{*}\left(\mathcal{O}_{\mathbb{P}^{n}}(s)\right)\right) \oplus\left(\pi_{1}^{*}\left(\mathcal{O}_{\mathbb{P}^{m}}(r)\right) \otimes_{\mathcal{O}_{X}} \pi_{2}^{*}\left(\Theta_{\mathbb{P}^{n}}(s)\right)\right),
$$

where we are assuming that both $r$ and $s$ are nonnegative integers. Denoting by $x_{1}, \ldots, x_{m+1}$ the homogeneous coordinates of $\mathbb{P}^{m}$ and by $y_{1}, \ldots, y_{n+1}$ those of $\mathbb{P}^{n}$, a section of the above sheaf can be written as an operator

$$
\xi=\sum_{i=1}^{m+1} a_{i} \frac{\partial}{\partial x_{i}}+\sum_{j=1}^{n+1} b_{j} \frac{\partial}{\partial y_{j}},
$$

where $a_{i}$ and $b_{j}$ are bihomogeneous polynomials of $\mathbb{C}\left[x_{1}, \ldots, x_{m+1}, y_{1}, \ldots, y_{n+1}\right]$ of bidegrees $(r+1, s)$ and $(r, s+1)$, respectively. In this form, the singularities of $\xi$ are the zeroes of the $2 \times 2$ minors of the matrices

$$
\left[\begin{array}{llll}
a_{1} & a_{2} & \cdots & a_{m+1} \\
x_{1} & x_{2} & \cdots & x_{m+1}
\end{array}\right] \text { and }\left[\begin{array}{llll}
b_{1} & b_{2} & \cdots & b_{n+1} \\
y_{1} & y_{2} & \cdots & y_{n+1}
\end{array}\right] \text {. }
$$

A closed set $Z$ of $\mathbb{P}^{m} \times \mathbb{P}^{n}$ is invariant under $\xi$ if and only if

$$
\xi(f) \in I(Z) \text { for all } f \in I(Z),
$$

where $I(Z)$ denotes the ideal of $Z$ in $\mathbb{C}\left[x_{1}, \ldots, x_{m+1}, y_{1}, \ldots, y_{n+1}\right]$. We finish with a proposition that relates closed sets invariant under $\xi$ with singularities of the foliation.

Proposition 3.1. Let $\xi$ be as above and let $Z$ be a closed subscheme invariant under $\xi$. If $\operatorname{Sing}(\xi)$ has codimension at least two in $\mathbb{P}^{m} \times \mathbb{P}^{n}$, then $\operatorname{Sing}(\xi) \cap Z \neq \emptyset$. 
Proof. The result will follow from [7, Proposition 5.3, p. 129] if we prove that $\omega_{\mathbb{P}^{m} \times \mathbb{P}^{n}}^{\vee} \otimes \mathcal{L}$ is ample, with $\mathcal{L}$ as in (3.1). But

$$
\omega_{\mathbb{P}^{m} \times \mathbb{P}^{n}}=\pi_{1}^{*} \mathcal{O}(-m-1) \otimes_{\mathcal{O}_{X}} \pi_{2}^{*} \mathcal{O}(-n-1)
$$

so that

$$
\omega_{\mathbb{P}^{m} \times \mathbb{P}^{n}}^{\vee} \otimes \mathcal{L} \cong \pi_{1}^{*} \mathcal{O}(m+1+r) \otimes_{\mathcal{O}_{X}} \pi_{2}^{*} \mathcal{O}(n+1+s) .
$$

Since $r$ and $s$ are nonnegative, the required hypothesis is verified and the proof is complete.

3.3. Notation. Let us now fix the notation that will be in force throughout the paper. Let

$$
\mathbb{C}[\mathbf{x}, \mathbf{y}]=\mathbb{C}\left[x_{1}, x_{2}, x_{3}, x_{4}, y_{1}, y_{2}, y_{3}\right] .
$$

We will also write $\mathbb{C}[\mathbf{x}]$ instead of $\mathbb{C}\left[x_{1}, x_{2}, x_{3}, x_{4}\right]$.

If $a, b, c \in \mathbb{C}[\mathbf{x}]$ are homogeneous polynomials of degree $k \geq 2$, then $f=a y_{1}+$ $b y_{2}+c y_{3}$ is bihomogeneous of bidegree $(k, 1)$ in $\mathbb{C}[\mathbf{x}, \mathbf{y}]$. Let

$$
d=a \frac{\partial}{\partial x_{1}}+b \frac{\partial}{\partial x_{2}}+c \frac{\partial}{\partial x_{3}} .
$$

Denote by $A, B$ and $C$ the derivatives of $f$ with respect to $x_{1}, x_{2}$ and $x_{3}$, and write

$$
\xi=d-\left(A \frac{\partial}{\partial y_{1}}+B \frac{\partial}{\partial x_{2}}+C \frac{\partial}{\partial x_{3}}\right) .
$$

Note that if $g \in \mathbb{C}[\mathbf{x}]$, then

$$
\xi(g)=d(g) .
$$

The vector field $\xi$ corresponds to the homogenization with respect to $x_{4}$ of the Hamiltonian vector field defined by the function $\left.f\right|_{x_{4}=1}$. Since $\xi$ is homogeneous in both the $x$ 's and $y$ 's, it induces a foliation in $\mathbb{P}^{3} \times \mathbb{P}^{2}$ which we also denote by $\xi$.

Now, if $g \in \mathbb{C}[\mathbf{x}, \mathbf{y}]$ is bihomogeneous, let $g_{0}$ be the bihomogeneous polynomial of $\mathbb{C}[\mathbf{x}, \mathbf{y}]$ obtained by setting $x_{4}$ equal to zero in $g$. Then,

$$
d_{0}=a_{0} \frac{\partial}{\partial x_{1}}+b_{0} \frac{\partial}{\partial x_{2}}+c_{0} \frac{\partial}{\partial x_{3}}
$$

induces a foliation in the plane (at infinity) $H_{\infty}$ defined by $x_{4}=0$, which corresponds to the restriction of $d$ to this projective plane. Similarly,

$$
\xi_{0}=d_{0}-\left(A_{0} \frac{\partial}{\partial y_{1}}+B_{0} \frac{\partial}{\partial x_{2}}+C_{0} \frac{\partial}{\partial x_{3}}\right)
$$

induces a foliation on the hyperplane $H_{\infty} \times \mathbb{P}^{2}$ of $\mathbb{P}^{3} \times \mathbb{P}^{2}$. As an immediate consequence of the definition of singularity in terms of minors of matrices given in \$3.2 we have that

$$
\pi_{1}\left(\operatorname{Sing}\left(\xi_{0}\right)\right)=\operatorname{Sing}\left(d_{0}\right)
$$

where $\pi_{1}: \mathbb{P}^{3} \times \mathbb{P}^{2} \rightarrow \mathbb{P}^{3}$, is the projection on the first component of the product. The next lemma contains three formulae that will be used throughout the paper without any further comment. As in the previous section $h=\sum_{i=1}^{3} x_{i} y_{i}$.

Lemma 3.2. If $p=\partial f / \partial x_{4}$, then

(1) $\xi(f)=0$;

(2) $\xi(h)=(1-k) f+x_{4} p$;

(3) $\xi_{0}(h)=(1-k) f_{0}$. 
Proof. (1) is obvious and (3) follows from (2); so we will prove only (2). By the definition of $\xi$ and $h$,

$$
\xi(h)=f-\left(A x_{1}+B x_{2}+C x_{3}\right) .
$$

Since $a, b$ and $c$ are homogeneous of degree $k$ with respect to the $x$ 's, we can apply Euler's relation to

$$
A x_{1}+B x_{2}+C x_{3}=\sum_{i=1}^{3} x_{i} \frac{\partial f}{\partial x_{i}},
$$

which gives

$$
A x_{1}+B x_{2}+C x_{3}=k f-x_{4} \frac{\partial f}{\partial x_{4}}=k f-x_{4} p .
$$

Hence,

$$
\xi(h)=f-(k f-p)=(1-k)+x_{4} p .
$$

We finish the section with a characterization of some subvarieties of the hypersurface $z(f)$.

Proposition 3.3. Every subvariety of codimension two of $\mathbb{P}^{3} \times \mathbb{P}^{2}$, contained in the hypersurface $Z(f)$, is a schematic complete intersection.

Proof. Suppose that $X$ is a subvariety of codimension two contained in the hypersurface $Z(f)$. Then, $f$ belongs to the ideal $\mathrm{I}(X)$. Since $X$ has codimension two in $\mathbb{P}^{3} \times \mathbb{P}^{2}$, it follows that the image of $\mathrm{I}(X)$ in the factor ring $S /(f)$ has codimension one. But this ring is factorial by Theorem 2.5. Therefore, $\mathrm{I}(X)$ is a principal ideal, and the proof is complete.

\section{Foliations of $\mathbb{P}^{n}$}

In this section we prove that when $n \geq 2$ a generic foliation of $\mathbb{P}^{n}$ which leaves $H_{\infty}$ invariant cannot have an invariant proper algebraic subvariety of positive dimension. Curves are handled separately because they require the methods of [7] and [14, while subvarieties of higher dimension can be dealt with by intersecting them with $H_{\infty}$. A similar result in dimension two has been proved in [3, Theorem 5, p. 242] and [15, Theorem 3, p. 385].

Lemma 4.1. Let $\chi \in \mathbb{Q}[t]$ and $k \geq 2$ be an integer. Given a point $p \in \mathbb{P}^{n}$, there exists a section of $\Theta_{\mathbb{P}^{n}}(k)$ which induces a foliation of $\mathbb{P}^{n}$, singular at $p$, none of whose invariant curves with Hilbert polynomial $\chi$ contain $p$.

Proof. The proof is essentially the same as that of [7, Proposition 4.1, p. 126]. Actually the only part of the proof that does not go through verbatim is the choice of foliation. That happens for two reasons. The first is that we are dealing here only with foliations that leave the hyperplane at infinity $H_{\infty}=\mathcal{Z}\left(x_{n+1}\right)$ invariant; the second is that the condition on the first cohomology group in the statement 7 , Proposition 4.1, p. 126] need not hold for lower powers of the line bundle $\mathcal{O}(1)$. We will overcome these problems by a direct computation, whose first step is a simple interpolation.

We begin by showing that, given points $p_{1}, p_{2} \in \mathbb{A}^{n}$, vectors $u_{1}, u_{2} \in \mathbb{C}^{n}$, complex numbers $c_{1}$ and $c_{2}$, and an integer $k \geq 2$, there exists a nonzero homogeneous polynomial $g \in \mathbb{C}\left[x_{1}, \ldots, x_{n+1}\right]$ such that

$$
g\left(p_{i}\right)=c_{i} \text { and } \nabla g\left(p_{i}\right)=u_{i}, \text { for } \quad 1 \leq i \leq 2 .
$$


In order to do this, choose $g$ to be a polynomial of degree $k$ in $\mathbb{C}\left[x_{1}, \ldots, x_{n+1}\right]$ with undetermined coefficients. The above equations give rise to 4 linear equations in the coefficients of $g$. Since a complete polynomial of degree $k$ in $n+1$ variables has more than four coefficients when $k \geq 2$, the resulting linear system always has a nonzero solution. So the required $g$ always exists.

Let us now apply this to derivations. Let $p_{1}$ and $p_{2}$ be points in the hyperplane $H_{\infty}=\mathcal{Z}\left(x_{n+1}\right)$ and $M_{1}, M_{2}$ be complex $n \times n$ matrices that stabilize this hyperplane. Note that this last condition implies that the last row of $M_{i}$ is $\left(0, \ldots, 0, m_{i}\right)$ for some complex number $m_{i}$. We may also assume, without loss of generality, that both $p_{1}$ and $p_{2}$ belong to the open set of $\mathbb{P}^{n}$ defined by $x_{1} \neq 0$. It follows from the preceding construction that there exists a homogeneous polynomial $a_{1} \in \mathbb{C}\left[x_{1}, \ldots, x_{n+1}\right]$ such that

$$
a_{1}\left(p_{i}\right)=m_{i} \text { and } \nabla a_{1}\left(p_{i}\right)=0, \text { for } 1 \leq i \leq 2 .
$$

Using the interpolation result again, we can now construct homogeneous polynomials $a_{2}, \ldots, a_{n} \in \mathbb{C}\left[x_{1}, \ldots, x_{n+1}\right]$ such that

$$
\left(a_{2}\left(p_{i}\right), \ldots, a_{n}\left(p_{i}\right)\right)=p_{i} m_{i} \text { and } J_{i}=M_{i}+m_{i} I, \text { for } 1 \leq i \leq 2,
$$

where $J_{i}$ denotes the jacobian of $\left(a_{2}, \ldots, a_{n}\right)$ relative to the variables $x_{2}, \ldots, x_{n}$, computed at the point $p_{i}$, and $I$ is the $n \times n$ identity matrix. A straightforward calculation shows that the foliation $\mathcal{F}$ of $\mathbb{P}^{n}$ induced by

$$
\sum_{i=1}^{n} a_{i} \frac{\partial}{\partial x_{i}}
$$

satisfies the following properties:

- $H_{\infty}$ is invariant under $\mathcal{F}$;

- $\mathcal{F}$ is singular at $p_{i}$

- the 1 -jet of $\mathcal{F}$ at $p_{i}$ is $M_{i}$;

for $1 \leq i \leq 2$. This is enough to prove that a foliation $\mathcal{F}$ with the properties required in the proof of [7, Proposition 4.1, p. 126] exists subject to two further conditions: $H_{\infty}$ is invariant under $\mathcal{F}$ and $p_{1}, p_{2} \in H_{\infty}$. As already pointed out, the remainder of the proof goes through with essentially no change.

Theorem 4.2. A generic algebraic foliation of $\mathbb{P}^{n}$ of degree $k \geq 2$ that leaves $H_{\infty}$ invariant does not have any algebraic invariant curves.

Proof. Let $\mathbb{C}[\mathbf{x}]=\mathbb{C}\left[x_{1}, \ldots, x_{n+1}\right]$ and denote by $\mathbb{C}[\mathbf{x}]_{k}$ its homogeneous component of degree $k$. Assume that $n>1$ and $k \geq 2$ are integers that will remain fixed throughout the proof. If $a_{i} \in \mathbb{C}[\mathbf{x}]_{k}$ for $1 \leq i \leq n$, then we identify the vector field

$$
d=\sum_{j=1}^{n} a_{i} \partial / \partial x_{i}
$$

with the $n$-tuple $\left(a_{1}, \ldots, a_{n}\right) \in\left(\mathbb{C}[\mathbf{x}]_{k}\right)^{n}$. Every foliation of degree $k$ over $\mathbb{P}^{n}$ which leaves the hyperplane $H_{\infty}$ of equation $x_{n+1}=0$ invariant can be defined by such a vector field. Indeed, we will use $d$ to denote both the vector field and the foliation that it determines in $\mathbb{P}^{n}$. Hence, these foliations are parameterized by the projective space $\Sigma=\mathbb{P}\left(\left(\mathbb{C}[\mathbf{x}]_{k}\right)^{n}\right)$. Note, however, that we are allowing for $\operatorname{gcd}\left(a_{1}, \ldots, a_{n}\right) \neq 1$, which gives rise to a nonsaturated foliation. To simplify the notation, $[d]$ will stand for the point $\left[a_{1}: \cdots: a_{n}\right] \in \Sigma$ that corresponds to the vector field $d$ as in (4.1). 
By [7. Lemma 5.2, p. 129] every subvariety invariant under a foliation of $\mathbb{P}^{n}$ must contain a singularity of this foliation. Therefore the foliation of $\mathbb{P}^{n}$ corresponding to a $[d] \in \Sigma$ must have a singularity at $H_{\infty}$. Let

$$
\mathcal{X}=\left\{[d] \times p \mid d \in \Sigma \text { and } p \in \operatorname{Sing}(d) \cap H_{\infty}\right\} .
$$

Let $q_{2}$ be the projection of $\mathcal{X}$ on $\mathbb{P}^{n}$ and note that the fibres $q_{2}^{-1}(p)$ are linear algebraic sets for every $p \in \mathbb{P}^{n}$. Therefore the fibres of $q_{2}$ are irreducible. Moreover, they have the same dimension because $\operatorname{PGL}(n)$ acts transitively on $\mathbb{P}^{n}$ and its action is compatible with $q_{2}$. Hence, $\mathcal{X}$ is irreducible.

For $\chi \in \mathbb{Q}[t]$ define $\mathcal{S}_{\chi}$, a subset of $\Sigma \times \mathbb{P}^{n}$, by

$\mathcal{S}_{\chi}=\{[d] \times p: p$ is in a subscheme, invariant under $d$, of Hilbert polynomial $\chi\}$.

Then, by [7, Proposition 2.1, p. 120], we have that $\mathcal{S}_{\chi}$ is a closed subset of $\Sigma \times \mathbb{P}^{n}$.

Assume now, by contradiction, that every $[d] \in \Sigma$ has an invariant curve with Hilbert polynomial $\chi$. Since the intersection of any such a curve with $H_{\infty}$ must contain a singularity of $d$, it follows that

$$
q_{1}\left(\mathcal{S}_{\chi} \cap \mathcal{X}\right)=\Sigma
$$

where $q_{1}$ denotes the restriction to $\mathcal{X}$ of the projection of $\Sigma \times \mathbb{P}^{n}$ on its first component. However, $\mathcal{X}$ is irreducible and its dimension is equal to that of $\Sigma$, because $q_{1}$ is generically finite. Thus,

$$
\mathcal{S}_{\chi} \cap X=X
$$

which implies that each curve invariant under $d$ must go through every point of $\operatorname{Sing}(d) \cap H_{\infty}$. But this contradicts Lemma 4.1 and completes the proof.

We may now proceed to the main theorem of this section.

Theorem 4.3. A generic algebraic foliation of $\mathbb{P}^{n}$ of degree $k \geq 2$ that leaves $H_{\infty}$ invariant cannot have any other proper algebraic invariant subvarieties of positive dimension.

Proof. Suppose that $d$ is such a generic foliation, and let $Y$ be a proper invariant subvariety of $d$. It follows from Theorem 4.2 that $\operatorname{dim}(Y)>1$. Thus,

$$
\operatorname{dim}\left(Y \cap H_{\infty}\right) \geq \operatorname{dim}(Y)-1>0
$$

by [11, Theorem 7.2, p. 48]. Hence, $\left.d\right|_{H_{\infty}}$ has an invariant subvariety of positive dimension contained in $H_{\infty}$. However, $H_{\infty} \cong \mathbb{P}^{n-1}$. Moreover, since $d$ is generic in $\mathbb{P}^{n}$, subject to the condition that it leaves $H_{\infty}$ invariant, its restriction to $H_{\infty}$ gives rise to a generic foliation of $\mathbb{P}^{n-1}$. But such a foliation cannot have any invariant proper algebraic subvarieties of positive dimension by [7, Theorem 1.1, p. 118] or [14, Theorem 2, p. 533]. Thus we have a contradiction, and the proof is complete.

\section{The Key LemMas}

5.1. Hypotheses. We begin the section by stating the hypotheses under which we are to work in the remainder of the paper. The notation of 3.3 remains in force. Recall that given a polynomial $g$ in

$$
\mathbb{C}[\mathbf{x}, \mathbf{y}]=\mathbb{C}\left[x_{1}, x_{2}, x_{3}, x_{4}, y_{1}, y_{2}, y_{3}\right] \quad \text { or } \quad \mathbb{C}[\mathbf{x}]=\mathbb{C}\left[x_{1}, x_{2}, x_{3}, x_{4}\right],
$$


we write $g_{0}=\left.g\right|_{x_{4}=0}$. Let $a, b$ and $c$ be homogeneous polynomials of degree $k \geq 2$ in $\mathbb{C}[\mathbf{x}]$. We will assume that:

H.1: $a_{0}, b_{0}$ and $c_{0}$ are nonzero;

H.2: $a_{0}$ is irreducible and the ideal $\left(a_{0}, b_{0}\right)$ is a radical ideal of $\mathbb{C}\left[x_{1}, x_{2}, x_{3}\right]$ of height 2 whose minimal primes do not contain $c_{0}$;

H.3: $(a, b)$ is a prime ideal;

H.4: $d_{0}$ has no invariant curves in $X=\mathbb{P}^{2}$;

H.5: $\xi_{0}$ does not have singularities in $Z\left(x_{3} y_{1}\right)$.

Note that (H.1) and (H.2) imply that $a$ is an irreducible homogeneous polynomial and that the ideal $(a, b)$ of $\mathbb{C}[\mathbf{x}]$ does not contain $c$. In particular, this implies that $\operatorname{Sing}(d)$ has dimension zero in $\mathbb{P}^{3}$.

5.2. The plane at infinity. Our first lemma is concerned with the behaviour of the foliation $\xi$ at infinity.

Lemma 5.1. Let $g_{0}$ be a bihomogeneous nonconstant polynomial contained in the ring $\mathbb{C}\left[x_{1}, x_{2}, x_{3}, y_{1}, y_{2}, y_{3}\right]$ such that $g_{0} \notin\left(f_{0}\right)$.

(1) If $\xi_{0}\left(g_{0}\right) \in\left(h, f_{0}, g_{0}\right)$, then $g_{0} \in\left(h, f_{0}\right)$.

(2) If $\xi_{0}\left(g_{0}\right) \in\left(f_{0}, g_{0}\right)$, then $g_{0} \equiv h^{m}\left(\bmod f_{0}\right)$. In particular, $g_{0}$ has bidegree $(m, m)$, for some integer $m>0$.

Proof. Let $A=\mathbb{C}\left[x_{1}, x_{2}, x_{3}, y_{1}, y_{2}, y_{3}\right]$. The hypothesis of (1), together with Lemma 3.2. imply that the ideal $I=\left(g_{0}, h, f_{0}\right)$ is invariant under $\xi_{0}$. But, by Propositions 2.3 and 2.4 and Theorem 2.5 $\left(f_{0}, h\right)$ is a prime ideal of height 2 contained in $I$. Hence, either $\left(f_{0}, h\right) \subsetneq I$ or $I=\left(f_{0}, h\right)$.

Taking into account that $f_{0}$ and $\left(f_{0}, h\right)$ are prime ideals, we have that if $\left(f_{0}, h\right) \subsetneq$ $I$, then

$$
\operatorname{dim}(Z(I))<\operatorname{dim}\left(Z\left(f_{0}, h\right)\right)=2 .
$$

Therefore, $Z(I)$ has dimension one or zero. In particular, if

$$
\pi_{1}: \mathbb{P}^{2} \times \mathbb{P}^{2} \rightarrow \mathbb{P}^{2}
$$

is the projection on the first component, then

$$
\operatorname{dim}\left(\pi_{1}(z(I))\right) \leq 1 .
$$

However, $\pi_{1}(\mathcal{Z}(I))$ is invariant under $d_{0}$, so it must have dimension zero by H.4. Let

$$
\pi_{1}(Z(I))=\left\{p_{1}, \ldots, p_{t}\right\} .
$$

If $p_{i}=\left[\alpha_{1}: \alpha_{2}: \alpha_{3}\right]$ and $\sigma$ is an element of the symmetric group $S_{3}$, let $g_{\sigma}^{i}=$ $\alpha_{\sigma(1)} x_{\sigma(2)}-\alpha_{\sigma(2)} x_{\sigma(1)}$. Hence, the ideal of $\pi_{1}(\mathcal{Z}(I))$ in $\mathbb{C}\left[x_{1}, x_{2}, x_{3}\right]$ is equal to the radical of $\mathfrak{m}_{1} \cdots \mathfrak{m}_{t}$, where $\mathfrak{m}_{i}=\left(g_{\sigma}^{i}: \sigma \in S_{3}\right)$ is a homogeneous maximal ideal of $\mathbb{C}\left[x_{1}, x_{2}, x_{3}\right]$ (properly contained in the irrelevant ideal) for $1 \leq i \leq t$. Thus, if

$$
\mu=g_{\sigma_{1}}^{1} \cdots g_{\sigma_{t}}^{t} \in \mathfrak{m}_{1} \cdots \mathfrak{m}_{t}
$$

for some choice of $\sigma_{1}, \ldots, \sigma_{t} \in S_{3}$, then there exists a positive integer $s>0$ such that

$$
\left(y_{j} \mu\right)^{s} \in I \text { for each } 1 \leq j \leq 3 .
$$

Moreover, since there are finitely many $\mu$ 's, we can choose one $m$ that will work for all of them. 
Now let $\beta=x_{3} b_{0}-x_{2} c_{0}$ and denote by $B$ the localization of the domain $A /\left(f_{0}, h\right)$, at the powers of $\beta x_{3}$. Since,

$$
x_{3} f_{0}-c_{0} h=\left(x_{3} a_{0}-c_{0} x_{1}\right) y_{1}+\beta y_{2},
$$

it follows that

$$
\beta y_{2}=x_{3} f_{0}-c_{0} h-\left(x_{3} a_{0}-c_{0} x_{1}\right) y_{1} \text { and that } x_{3} y_{3}=h-\left(x_{1} y_{1}-x_{2} y_{2}\right) .
$$

Hence

$$
B \cong\left(\mathbb{C}\left[x_{1}, x_{2}, x_{3}\right]_{\beta x_{3}}\right)\left[y_{1}\right],
$$

which is a factorial ring by [16, Théoréme 4, p. 29]. Moreover, each $\mathfrak{m}_{i}$ has two distinct linear generators, so we can choose

$$
\mu, \mu^{\prime} \in \mathfrak{m}_{1} \cdots \mathfrak{m}_{t},
$$

with $\operatorname{gcd}\left(\mu, \mu^{\prime}\right)=1$ in $B$. But, by (5.1),

$$
y_{1}^{m} \mu^{m}, y_{1}^{m} \mu^{\prime m} \in B I,
$$

where $B I$ is the extension of $I$ to $B$. Denoting by $\overline{g_{0}}$ the image of $g_{0}$ in $A /\left(f_{0}, h\right)$, we conclude that $B I=B \overline{g_{0}}$ because $\overline{f_{0}}=\bar{h}=\overline{0}$. Thus, $\overline{g_{0}}$ divides both ${\overline{y_{1}}}^{m} \bar{\mu}^{m}$ and $\overline{y_{1}} \bar{\mu}^{\prime}{ }^{m}$, so it must divide their greatest common divisor, which is ${\overline{y_{1}}}^{m}$. However, $\overline{y_{1}}$ is a prime in $B$, which implies that

$$
\overline{g_{0}}=u{\overline{y_{1}}}^{k}, \text { for some unit } u \text { in } B .
$$

Note that it follows from (5.2) that the units of $B$ are products of powers of $x_{3}$ and $\beta$ with integral exponents.

Now we analyse the various possible identities over $A /\left(f_{0}, h\right)$ that result from (5.3), also taking into account the explicit description of units of $B$ given above. Throughout this discussion $k, \ell$ and $m$ denote nonnegative integers and $\bar{a}$ denotes the class of $a \in A$ in the ring $A /\left(f_{0}, h\right)$. One of the four possible identities, namely

$$
\bar{\beta}^{m}{\overline{x_{3}}}_{\ell}{\overline{g_{0}}}={\overline{y_{1}}}^{k}
$$

can be eliminated straightaway because $A$ is a bigraded ring and the degree in the $x$ 's do not match on the two sides of the equation. Thus, by the bihomogeneity of $g_{0}$, it follows that $g_{0}=y_{1}^{k}$, which will be analysed later on as part of the last case. The next identity we consider is

$$
\bar{\beta}^{m}{\overline{g_{0}}}^{=}{\overline{x_{3}}}^{\ell}{\overline{y_{1}}}^{k} .
$$

Let $X$ be an irreducible component of $z\left(f_{0}, h, g_{0}\right)$ and $P$ be its associated prime ideal. By (5.4), $P$ contains either $x_{3}$ or $y_{1}$. Therefore,

$$
X \subset z\left(x_{3}\right) \cup z\left(y_{1}\right) .
$$

However, $X$ must contain a singularity of $\xi_{0}$ by Proposition 3.1, so we have a contradiction with hypothesis H.5.

The third possibility is

$$
\bar{x}_{3} \ell \bar{g}_{0}=\bar{\beta}^{m} \bar{y}^{k}
$$

which implies that $x_{2} \widetilde{c_{0}} y_{1} \in \sqrt{\left(f_{0}, h, x_{3}\right)}$, where $\widetilde{c_{0}}=c_{0}\left(x_{1}, x_{2}, 0\right)$. Thus,

$$
R=A /\left(f_{0}, h, x_{3}\right)
$$


will vanish when localized at $x_{2} \widetilde{c_{0}} y_{1}$. However,

$$
\left(x_{3}, y_{2}+\frac{x_{1}}{x_{2}} y_{1}, y_{3}+\left(\frac{\widetilde{b_{0}} x_{1}}{\widetilde{c_{0}} x_{1}}-\frac{\widetilde{a_{0}}}{\widetilde{c_{0}}}\right) y_{1}\right)=\left(f_{0}, h, x_{3}\right)_{x_{2} \widetilde{c_{0}} y_{1}} \text { in } A_{x_{3} \widetilde{c_{0}} y_{1}}
$$

so that

$$
R_{x_{2} \widetilde{c_{0} y_{1}}} \cong\left(\mathbb{C}\left[x_{1}, x_{2}\right]_{x_{2} \widetilde{c_{0}}}\left[y_{1}\right]\right)_{y_{1}} \neq 0,
$$

which is a contradiction. Finally, we have

$$
\overline{g_{0}}=\bar{\beta}^{m}{\overline{x_{3}}}_{\ell}{\overline{y_{1}}}^{k} .
$$

If $k=0$, then $g_{0} \in \mathbb{C}\left[x_{1}, x_{2}, x_{3}\right]$, which implies that

$$
\left(f_{0}, h, g_{0}\right) \cap \mathbb{C}\left[x_{1}, x_{2}, x_{3}\right]=\left(g_{0}\right)
$$

is invariant under $d_{0}$, contradicting H.4. Hence, $k>0$ and

$$
\left(f_{0}, h, g_{0}\right) \subseteq\left(f_{0}, h, y_{1}\right) .
$$

However, by (5.1), there exists an integer $s>0$ such that

$$
\left(\mu y_{3}\right)^{s} \in\left(f_{0}, h, g_{0}\right) \text { for every } \mu \in \mathfrak{m}_{1} \cdots \mathfrak{m}_{t} .
$$

Combined with (5.5) this implies that

$$
\left(\mu y_{3}\right)^{s}=\phi_{1} f_{0}+\phi_{2} h+\phi_{3} y_{1}
$$

for bihomogeneous polynomials $\phi_{1}, \phi_{2}, \phi_{3} \in A$. Taking $y_{1}=y_{2}=0$ into (5.6) we end up with

$$
\left(\mu y_{3}\right)^{s}=\left(\widetilde{\phi_{1}} c_{0}+\widetilde{\phi_{2}} x_{3}\right) y_{3} \text {, where } \widetilde{\phi_{j}}=\left.\left(\phi_{j}\right)\right|_{y_{1}=y_{2}=0} .
$$

Therefore,

$$
\mu^{s} \in\left(c_{0}, x_{3}\right) \text { for every } \mu \in \mathfrak{m}_{1} \cdots \mathfrak{m}_{t},
$$

which implies that

$$
\mathfrak{m}_{1} \cdots \mathfrak{m}_{t} \subseteq \sqrt{\left(c_{0}, x_{3}\right)} .
$$

Hence,

$$
\emptyset \neq z\left(c_{0}, x_{3}\right) \subseteq \mathcal{Z}\left(\mathfrak{m}_{1} \cdots \mathfrak{m}_{t}\right) \subseteq \operatorname{Sing}\left(d_{0}\right) .
$$

But this means that there are singularities of $d_{0}$ contained in $z\left(x_{3}\right)$. Taken together with (3.2), this contradicts H.5. Therefore, $\left(f_{0}, h\right) \subsetneq I$ cannot occur, and we conclude that $I=\left(f_{0}, h\right)$. In particular, $g_{0} \in\left(f_{0}, h\right)$, which proves $(1)$.

Assume now that $\xi_{0}\left(g_{0}\right) \in\left(f_{0}, g_{0}\right)$. By $(1)$ we conclude that $g_{0} \equiv q h^{m}\left(\bmod f_{0}\right)$ for some polynomial $q$ and some integer $m>0$. Moreover, as $A /\left(f_{0}\right)$ is a factorial domain by Theorem 2.5. we can assume that $q$ is coprime with $h$ modulo $f_{0}$. If $q$ is constant, the result is proved, so we assume that $q$ is nonconstant and aim at a contradiction. By Lemma 3.2,

$$
\xi_{0}\left(g_{0}\right) \equiv \xi_{0}(q) h^{m}+q m h^{m-1} \xi_{0}(h) \equiv \xi_{0}(q) h^{m} \quad\left(\bmod f_{0}\right) .
$$

Taking into account that $g_{0}$ is invariant under $\xi_{0}$ modulo $f_{0}$, we conclude that

$$
\xi_{0}(q) \equiv r q \quad\left(\bmod f_{0}\right),
$$

for some $r \in A$. But this implies that $\xi_{0}(q) \in\left(h, f_{0}, q\right)$, so $q \in\left(h, f_{0}\right)$ by part (1), which contradicts the choice of $q$ and completes the proof. 
5.3. Ideals of codimension two. From now on $g$ will denote a bihomogeneous nonconstant polynomial of $\mathbb{C}[\mathbf{x}, \mathbf{y}]$ that is not contained in $\left(x_{4}\right)$. Moreover, we will assume that $(f, g)$ is a prime ideal of codimension 2 and invariant under $\xi$.

Lemma 5.2. If $(f, g)$ is invariant under $\xi$, then

$$
p_{0} \in(h)+\mathbb{C} A_{0}+\mathbb{C} B_{0}+\mathbb{C} C_{0} .
$$

Proof. By Lemma 5.1, $g$ can be written in the form

$$
g=h^{m}+x_{4} q,
$$

for some $q \in \mathbb{C}[\mathbf{x}, \mathbf{y}]$ and some integer $m>0$. Note that $q$ has bidegree $(m-1, m)$. Now, it follows from Lemma 3.2 that

$$
\xi(g)=m(1-k) h^{m-1} f+x_{4}\left(\xi(q)+m h^{m-1} p\right) .
$$

However, since $(f, g)$ is invariant under $\xi$,

$$
\xi(g) \equiv r g \quad(\bmod f)
$$

for some $r \in \mathbb{C}[\mathbf{x}]$. Hence,

$$
x_{4}\left(\xi(q)+m h^{m-1} p\right) \equiv r g \quad(\bmod f) .
$$

By Theorem 2.5, the ring $\mathbb{C}[\mathbf{x}, \mathbf{y}] /(f)$ is a factorial domain over which $x_{4}$ is irreducible. Since $x_{4}$ does not divide $h$, it cannot divide $g$ modulo $f$. Therefore, $x_{4}$ divides $r$ modulo $f$; say $r \equiv x_{4} \hat{r}(\bmod f)$ for some $\hat{r} \in \mathbb{C}[\mathbf{x}]$ of degree $k-2$. Cancelling $x_{4}$ in (5.7) we obtain

$$
\xi(q)+m h^{m-1} p \equiv \hat{r} g \quad(\bmod f) .
$$

Thus, using the convention of $₫ 3.3$ that a zero subscript indicates restriction to the hyperplane $H_{\infty}$ with equation $x_{4}=0$, we have

$$
\xi_{0}\left(q_{0}\right) \equiv h^{m-1}\left(\hat{r}_{0} h-m p_{0}\right) \quad\left(\bmod \left(f, x_{4}\right)\right),
$$

which implies that the ideal $\left(q_{0}, h, f_{0}\right)$ of $\mathbb{C}[\mathbf{x}, \mathbf{y}]$ is invariant under $\xi_{0}$. Thus, $q_{0} \in$ $\left(h, f_{0}\right)$ by Lemma 5.1, so that

$$
q_{0} \equiv h^{s} \widehat{q_{0}} \quad\left(\bmod f_{0}\right)
$$

for some integer $s>0$ and some polynomial $q_{0} \in A=\mathbb{C}\left[x_{1}, x_{2}, x_{3}, y_{1}, y_{2}, y_{3}\right]$. Moreover, since the ring $A /\left(f_{0}\right)$ is a factorial domain by Theorem 2.5, we can assume that $h$ and $\widehat{q_{0}}$ have no common factor module $f_{0}$. However, from (5.8) and (5.9) it follows that

$$
h^{s} \xi_{0}\left(\widehat{q_{0}}\right) \equiv h^{m-1}\left(\hat{r}_{0} h-m p_{0}\right) \quad\left(\bmod f_{0}\right) .
$$

If $\widehat{q_{0}}$ is a constant, then $\hat{r}_{0} h-m p_{0}$ must be divisible by $f_{0}$. However, $\hat{r}_{0} h-m p_{0}$ has degree $k-1$ with respect to the $x$ 's, while $f_{0}$ has degree $k$ relative to the same variables. This implies that $\hat{r}_{0} h=m p_{0}$, from which we conclude that $p_{0} \in(h)$, because $m \geq 1$. So we may assume that $q_{0}$ is not a constant.

Thus, either $s>m-1$ or $s \leq m-1$. In the first case, $h$ divides $p_{0}$ and we are done; in the second case we get that

$$
\xi_{0}\left(\widehat{q_{0}}\right) \equiv h^{m-s-1}\left(\hat{r}_{0} h-m p_{0}\right) \quad\left(\bmod f_{0}\right) .
$$

If $s<m-1$, then the ideal $\left(h, f_{0}, \widehat{q_{0}}\right)$ is invariant under $\xi_{0}$. But, by Lemma 5.1 this implies that $h$ divides $\widehat{q_{0}}$, contradicting the choice of $\widehat{q_{0}}$. Therefore, $s=m-1$ and

$$
\xi_{0}\left(\widehat{q_{0}}\right) \equiv \hat{r}_{0} h-m p_{0} \quad\left(\bmod f_{0}\right) .
$$


Since $q_{0}$ has bidegree $(m-1, m)$ and

$$
q_{0} \equiv h^{m-1} \widehat{q_{0}} \quad\left(\bmod f_{0}\right),
$$

by (5.9), it follows that $\widehat{q_{0}}$ has bidegree $(0,1)$. Hence, there exist complex numbers $e_{1}, e_{2}$ and $e_{3}$ such that

$$
\widehat{q_{0}}=e_{1} y_{1}+e_{2} y_{2}+e_{3} y_{3}
$$

so

$$
\xi_{0}\left(\widehat{q_{0}}\right) \equiv e_{1} A_{0}+e_{2} B_{0}+e_{3} C_{0} \quad\left(\bmod f_{0}\right) .
$$

Comparing with (5.11) we find that

$$
m p_{0} \equiv \hat{r}_{0} h-\left(e_{1} A_{0}+e_{2} B_{0}+e_{3} C_{0}\right) \quad\left(\bmod f_{0}\right) .
$$

However, $f_{0}$ has degree $k \geq 2$ with respect to the $x$ 's, so the congruence can only hold if

$$
m p_{0}=\hat{r}_{0} h-\left(e_{1} A_{0}+e_{2} B_{0}+e_{3} C_{0}\right),
$$

and the proof is complete.

\section{The MAIN Results}

The notation of $₫ 3.3$ and the hypotheses of $\$ 5.1$ will remain in force throughout this section.

\subsection{Invariant algebraic varieties. Let}

$$
\pi_{1}: \mathbb{P}^{3} \times \mathbb{P}^{2} \rightarrow \mathbb{P}^{3}
$$

be the projection on the first component. We begin with a theorem that characterizes the algebraic varieties invariant under the foliation $\xi$ of $\mathbb{P}^{3} \times \mathbb{P}^{2}$.

Theorem 6.1. Let $X \subsetneq H_{\infty} \times \mathbb{P}^{2}$ be a proper algebraic subvariety of positive dimension of the hypersurface $Z(f) \subset \mathbb{P}^{3} \times \mathbb{P}^{2}$ invariant under $\xi$, and suppose that the hypotheses of $\$ 5.1$ are satisfied. If

(1) $p_{0} \notin(h)+\mathbb{C} A_{0}+\mathbb{C} B_{0}+\mathbb{C} C_{0}$, then $\operatorname{dim}(X)<3$. If, moreover,

(2) all the algebraic curves and surfaces of $\mathbb{P}^{3}$ invariant under $d$ are contained in $H_{\infty}$,

then $X$ is contained in a finite union of fibres of $\pi_{1}$.

Proof. Let $X$ be an algebraic subvariety of $z(f)$ invariant under $\xi$. If $\operatorname{dim}(X)=3$, then, by Proposition 3.3. the ideal $\mathrm{I}(X)$ is generated by $f$ and by a bihomogeneous polynomial $g$. But by Lemma 5.2 this implies that

$$
p_{0} \in(h)+\mathbb{C} A_{0}+\mathbb{C} B_{0}+\mathbb{C} C_{0},
$$

which contradicts $(1)$. Therefore, $\operatorname{dim}(X)<3$. Hence,

$$
\operatorname{dim}\left(\pi_{1}(X)\right) \leq \operatorname{dim}(X) \leq 2 .
$$

Since $d$ has no invariant curves or surfaces, it follows that $\pi_{1}(X)$ is a set of isolated points, and the theorem follows.

Corollary 6.2. Let $k \geq 3$ and let $f$ be a bihomogeneous generic polynomial of bidegree $(k, 1)$ in $\mathbb{C}\left[x_{1}, x_{2}, x_{3}, x_{4}, y_{1}, y_{2}, y_{3}\right]$. If $X$ is an irreducible algebraic subvariety of codimension one or two in the hypersurface $\mathcal{Z}(f)$ of $\mathbb{P}^{3} \times \mathbb{P}^{2}$ that is invariant under $\xi$, then $X$ is contained in either $H_{\infty} \times \mathbb{P}^{2}$ or a fibre of $\pi_{1}$. 
Proof. We begin by showing that the hypotheses of Theorem 6.1 hold generically. Hypotheses H.1 and H.5 are clearly satisfied by a generic choice of $a, b$ and $c$, because the homogeneous components of $\mathbb{C}\left[x_{1}, x_{2}, x_{3}\right]$ of degree $k \geq 2$ have dimension greater than 3. The first part of H.2 is also clear. For the second part, let $\mathfrak{m}$ be a maximal ideal corresponding to $p \in \mathcal{Z}\left(a_{0}, b_{0}\right)$. Then, $c_{0} \in \mathfrak{m}$ if and only if $c_{0}(p)=0$, which is itself equivalent to

$$
z\left(a_{0}, b_{0}\right) \cap z\left(c_{0}\right) \neq \emptyset
$$

which does not hold for a generic choice of $a_{0}, b_{0}$ and $c_{0}$. Thus, a minimal prime $\mathfrak{m}$ over $\left(a_{0}, b_{0}\right)$ does not contain $c_{0}$. In order to prove H.3, we use the NoetherLefschetz Theorem, according to which the Picard group of the surface $S \subset \mathbb{P}^{3}$, with equation $a=0$, is generated by the hyperplane bundle when $k \geq 3$ and $a$ is chosen generically; see [8, Théoréme 1.2]. Since $S$ is a complete intersection, it is also projectively normal. Therefore, its homogeneous coordinate ring $\mathbb{C}[\mathbf{x}] / a$ is a factorial ring, [11, Exercise 6.3(c), p. 147]. Thus, any generically chosen element $b$ of degree $k$ in $\mathbb{C}[\mathbf{x}] / a$ will be prime. In particular, $(a, b)$ is a prime ideal. This proves that H.3 holds generically. The genericity of H.4 is a famous result of Jouanolou; see [13. Théoréme 1.1, p. 158]. Let us now turn to hypotheses (1) and (2) of Theorem 6.1 .

In order to simplify the notation, let $S(r, t)$ be the $\mathbb{C}$-vector subspace of bihomogeneous polynomials of bidegree $(r, t)$ in $\mathbb{C}\left[x_{1}, x_{2}, x_{3}, y_{1}, y_{2}, y_{3}\right]$. Since $p_{0}$ can be chosen independently of $A_{0}, B_{0}$ and $C_{0}$, it is enough to show that the vector space quotient

$$
\frac{S(k-1,1)}{S(k-2,0) h+\mathbb{C} A_{0}+\mathbb{C} B_{0}+\mathbb{C} C_{0}}
$$

is nonzero. However, this space has dimension greater than or equal to

$$
3\left(\begin{array}{c}
k+2 \\
2
\end{array}\right)-\left(\begin{array}{c}
k+1 \\
2
\end{array}\right)-3=\frac{2 k^{2}+8 k}{2}>0
$$

for all $k \geq 2$, so it must be nonzero, and we have (1). Finally, (2) is a consequence of Theorem 4.3

\section{A conjecture of Bernstein and Lunts}

The conjecture of Bernstein and Lunts for dimension three is a consequence of Theorem 6.1. We begin by reviewing the background of the conjecture.

7.1. Involutive varieties. Let $\partial_{i}$ denote the partial differential operator $\partial / \partial x_{i}$. For each multi-index $\alpha=\left(\alpha_{1}, \ldots, \alpha_{n}\right) \in \mathbb{N}^{n}$ we write

$$
\partial^{\alpha}=\partial_{1}^{\alpha_{1}} \cdots \partial_{n}^{\alpha_{n}}, \text { and }|\alpha|=\alpha_{1}+\cdots+\alpha_{n} .
$$

Using this notation, a polynomial differential operator over $\mathbb{C}^{n}$ can be written in the form

$$
P=\sum_{\alpha \in \mathcal{A}} a_{\alpha} \partial^{\alpha},
$$

where $a_{\alpha} \in \mathbb{C}\left[x_{1}, \ldots, x_{n}\right]$ and $a_{\alpha}=0$ for all multi-indices outside a finite set $\mathcal{A}$. The order of $P$ is $s$ if $|\alpha| \leq s$ for all $\alpha \in \mathcal{A}$ and $|\alpha|=s$ for at least one $\alpha$ for which $a_{\alpha} \neq 0$. The set of differential operators over $\mathbb{C}^{n}$ with the standard addition and multiplication (composition of operators) is a noncommutative ring, called the $n$th Weyl algebra and denoted by $A_{n}$. 
The order of an operator determines a filtration $\left\{F_{i}\right\}_{i \geq 0}$ in $A_{n}$, from which we can construct a graded ring. If $F_{i}$ is the complex vector space of all operators of $A_{n}$ of order less than or equal to $i$, then $F_{i} / F_{i-1}$ is isomorphic to the free $\mathbb{C}\left[x_{1}, \ldots, x_{n}\right]$ module $S_{i}$ generated by the monomials $y^{\alpha}$ with $|\alpha|=i$. Thus, one has a $\mathbb{C}$-linear map

$$
\sigma_{i}: F_{i} / F_{i-1} \rightarrow S_{i}
$$

called the symbol map of order $i$. We will write $y_{i}$ for the symbol $\sigma_{1}\left(\partial_{i}\right)$. If $P \in A_{n}$ has order $i$, then its principal symbol is

$$
\sigma(P)=\sigma_{i}(P)=\sum_{\substack{\alpha \in \mathcal{A} \\|\alpha|=i}} a_{\alpha} y^{\alpha} \in S_{i} .
$$

This grading induces an isomorphism of graded $\mathbb{C}\left[x_{1}, \ldots, x_{n}\right]$-algebras between the polynomial ring $\mathbb{C}\left[x_{1}, \ldots, x_{n}, y_{1}, \ldots, y_{n}\right]$ and $\bigoplus_{i \geq 0} S_{i}$ which, in turn, is naturally isomorphic to the coordinate ring of the cotangent bundle $T^{*} \mathbb{C}^{n}$. We will use $S$ to denote any of these three isomorphic rings.

Given a left ideal $J$ of $A_{n}$, its symbol ideal is the ideal of $S$ generated by $\sigma(P)$ for every $P \in J$. The construction of $\sigma(J)$ implies that the variety $\mathcal{Z}(\sigma(J))$ defined in $T^{*} \mathbb{C}^{n}$ by the vanishing of the elements of $\sigma(J)$ is subject to certain important constraints. First of all, $z(\sigma(J))$ is conical; that is, homogeneous with respect to the fibres of the cotangent bundle. Second, if $z(\sigma(J))$ does not contain the zero section of the cotangent bundle, then $J$ must have an operator of order zero (a polynomial of $\left.\mathbb{C}\left[x_{1}, \ldots, x_{n}\right]\right)$. The third, and most important, of the properties of $z(\sigma(J))$ is related to the fact that the cotangent bundle of a manifold always admits a symplectic structure; see [2, p. 202].

In the case of $T^{*} \mathbb{C}^{n}$ the symplectic structure is defined by the 2 -form

$$
\sum_{i=1}^{n} d x_{i} \wedge d y_{i}
$$

which, in turn, determines a Poisson bracket in $S$ given by the formula

$$
\{f, g\}=\sum_{i=1}^{n}\left(\frac{\partial f}{\partial x_{i}} \frac{\partial g}{\partial y_{i}}-\frac{\partial g}{\partial x_{i}} \frac{\partial f}{\partial y_{i}}\right),
$$

where $f, g \in S=\mathbb{C}\left[x_{1}, \ldots, x_{n}, y_{1}, \ldots, y_{n}\right]$. An ideal $I$ of $S$ is closed under the Poisson bracket if $\{I, I\} \subset I$. A conical variety whose ideal is closed under the Poisson bracket is called involutive. Such a variety must have dimension at least $n$; see [6, Proposition 2.2, p. 102] for a proof. The third property of $\mathcal{Z}(\sigma(J))$ can now be stated.

Theorem 7.1. Let $J$ be a left ideal of $A_{n}$. The radical of $\sigma(J)$ is closed under the Poisson bracket of $S$. In other words, $Z(\sigma(J))$ is an involutive conical variety.

This result, often referred to as the involutivity (or integrability) of the characteristic variety is far subtler than it seems at first. See [6, Chapter 11] for more details and [10] for a purely algebraic proof of the theorem.

We will apply these constructions to the special case in which the left ideal $J$ is generated by a first-order differential operator $P \in A_{n}$. In this case, $\sigma(J)=$ $(\sigma(P))$, so $\boldsymbol{Z}(\sigma(J))=\mathcal{Z}(\sigma(P))$ is a hypersurface of $T^{*} \mathbb{C}^{n}$. Moreover, $\mathcal{Z}(\sigma(P))$ always contains the zero section of $T^{*} \mathbb{C}^{n}$ as well as all the fibres that are supported over singularities of $P$. Bernstein and Lunts [3, §4.2, p. 236] defined an involutive conical 
variety of $T^{*} \mathbb{C}^{n}$ to be almost minimal if its only conical involutive subvarieties are the zero section of $T^{*} \mathbb{C}^{n}$ and a finite number of fibres. They used this concept in order to prove the following result; see [3, Proposition 6, p. 237].

Proposition 7.2. Let $P$ be a derivation of $\mathbb{C}\left[x_{1}, \ldots, x_{n}\right]$. If $\mathcal{Z}(\sigma(P))$ is almost minimal, then there exists a polynomial $f$ such that the left ideal $A_{n}(P+f)$ is maximal.

7.2. The conjecture. Of course for Proposition 7.2 to be meaningful one must first prove that there exist first-order polynomial operators whose characteristic varieties are almost minimal involutive. In [3, Conjecture 1, p. 236], Bernstein and Lunts proposed the following conjecture.

Conjecture 7.3. If $P$ is a generic derivation of $\mathbb{C}\left[x_{1}, \ldots, x_{n}\right]$ whose coefficients have degree $k \geq 2$, then $Z(\sigma(P))$ is an almost minimal conical involutive variety.

In [3, $\$ 4.3$, p. 236], they proved that the conjecture holds for $n=2$. In this case, the proof is quite simple, because if $\mathcal{Z}(\sigma(P))$ were not almost minimal, then it would contain a conormal subvariety supported on a curve, which would have to be invariant under $P$. But a generic derivation does not have any invariant curves by Theorem 4.3. We use the results of this paper to give a proof of the conjecture when $n=3$.

Theorem 7.4 (Conjecture of Bernstein and Lunts in dimension 3). If $P$ is a generic derivation whose coefficients are polynomials of degree $k \geq 3$ in $\mathbb{C}\left[x_{1}, x_{2}, x_{3}\right]$, then the hypersurface $\sigma(P)=0$ is almost minimal involutive in $T^{*} \mathbb{C}^{3}$.

Proof. Given an irreducible conical algebraic subvariety $X$ of $T^{*} \mathbb{C}^{3} \cong \mathbb{C}^{3} \times \mathbb{C}^{3}$ that is not contained in the zero section, we can construct an algebraic subvariety $\bar{X}$ of $\mathbb{P}^{3} \times \mathbb{P}^{2}$ via the standard embedding $\mathbb{C}^{3} \subset \mathbb{P}^{3}$. The homogenizing variable will be denoted by $x_{4}$. Applying this construction to the hypersurface $\sigma(P)=0$ we obtain the hypersurface $Z(f)$, where $f$ is the homogenization of $\sigma(P)$ with respect to $x_{4}$.

If $X$ is a subvariety of the hypersurface $\sigma(P)=0$ that is not contained in the zero section, then $\bar{X}$ is a subvariety $z(f)$ that is not contained in $H_{\infty} \times \mathbb{P}^{2}$. Moreover, if $X$ is involutive, then it is invariant under the Hamiltonian vector field induced by $f$. But this is equivalent to saying that $\bar{X}$ is invariant under $\xi$ in the notation of 33.3 . Thus, by Corollary $6.2, \bar{X}$ must be contained in a fibre of the projection

$$
\pi: \mathbb{P}^{3} \times \mathbb{P}^{2} \rightarrow \mathbb{P}^{3},
$$

which implies that $X$ is contained in a fibre of the cotangent bundle. Hence, $\sigma(P)=0$ is almost minimal involutive.

\section{REFERENCES}

1. W. W. Adams and P. Loustaunau, An introduction to Gröbner bases, Graduate Studies in Mathematics, vol. 3, American Mathematical Society, Providence, 1994. MR 1287608 (95g:13025)

2. V. I. Arnol'd, Mathematical methods of classical mechanics, Graduate Texts in Mathematics, vol. 60, Springer-Verlag, New York, 1989. Translated from the 1974 Russian original by K. Vogtmann and A. Weinstein, second edition. MR.997295 (90c:58046)

3. J. Bernstein and V. Lunts, On non-holonomic irreducible D-modules, Invent. Math. 94 (1988), 223-243. MR958832 (90b:58247)

4. A. Borel, P.-P. Grivel, B. Kaup, A. Haefliger, B. Malgrange, and F. Ehlers, Algebraic Dmodules, Perspectives in Mathematics, vol. 2, Academic Press Inc., Boston, MA, 1987. MR 882000 (89g:32014) 
5. Neil Chriss and Victor Ginzburg, Representation theory and complex geometry, Birkhäuser Boston Inc., Boston, MA, 1997. MR1433132 (98i:22021)

6. S. C. Coutinho, A primer of algebraic D-modules, London Mathematical Society Student Texts, vol. 33, Cambridge University Press, Cambridge, 1995. MR1356713 (96j:32011)

7. S. C. Coutinho and J. V. Pereira, On the density of algebraic foliations without algebraic invariant sets, J. Reine Angew. Math. 594 (2006), 117-135. MR2248154 (2007i:14010)

8. P. Deligne, Le théorème de noether, Groupes de monodromie en géométrie algébrique II, Séminaire de Géométrie Algébrique du Bois-Marie 1967-1969, (SGA 7 II). Dirigé par P. Deligne et N. Katz, Lecture Notes in Math., vol. 340, Springer, Berlin, 1973, pp. 328-340. MR 0354657 (50:7135)

9. David Eisenbud, Commutative algebra, With a view toward algebraic geometry, Graduate Texts in Mathematics, vol. 150, Springer-Verlag, New York, 1995. MR.1322960 (97a:13001)

10. Ofer Gabber, The integrability of the characteristic variety, Amer. J. Math. 103 (1981), no. 3, 445-468. MR618321 (82j:58104)

11. Robin Hartshorne, Algebraic geometry, Graduate Texts in Mathematics, No. 52, SpringerVerlag, New York, 1977. MR0463157 (57:3116)

12. Ryoshi Hotta, Kiyoshi Takeuchi, and Toshiyuki Tanisaki, D-modules, perverse sheaves, and representation theory, Progress in Mathematics, vol. 236, Birkhäuser Boston Inc., Boston, MA, 2008. Translated from the 1995 Japanese edition by Takeuchi. MR 2357361 (2008k:32022)

13. J. P. Jouanolou, Equations de Pfaff algébriques, Lect. Notes in Math., vol. 708, SpringerVerlag, Heidelberg, 1979. MR 537038 (81k:14008)

14. Valery Lunts, Algebraic varieties preserved by generic flows, Duke Math. J. 58 (1989), no. 3, 531-554. MR1016433 (91a:32015)

15. A. Lins Neto, P. Sad, and B. Scárdua, On topological rigidity of projective foliations, Bull. Soc. Math. France 126 (1998), 381-406. MR1682801 (2000b:32027)

16. P. Samuel, Anneaux factoriels, Rédaction de Artibano Micali, Sociedade de Matemática de São Paulo, São Paulo, 1963. MR0156867 (28:110)

17. J. T. Stafford, Nonholonomic modules over Weyl algebras and enveloping algebras, Invent. Math. 79 (1985), no. 3, 619-638. MR782240 (86h:17009)

Departamento de Ciência da Computação, Instituto de Matemática, Universidade Federal do Rio de Janeiro, P.O. Box 68530, 21945-970 Rio de Janeiro, Rio de Janeiro, Brazil - and - Programa de Engenharia de Sistemas e Computação, CopPe, Universidade Federaldo Rio de Janeiro, PO Box 68511, 21941-972 Rio de Janeiro, Rio de Janeiro, BRAZIL

E-mail address: collier@impa.br

URL: http://www.dcc.ufrj.br/〜 collier 